\title{
DCLK1 Regulates Pluripotency and Angiogenic Factors via microRNA-Dependent Mechanisms in Pancreatic Cancer
}

\author{
Sripathi M. Sureban ${ }^{1,4,5}$, Randal May ${ }^{1,4}$, Dongfeng $Q u^{1,4}$, Nathaniel Weygant ${ }^{1}$, Parthasarathy \\ Chandrakesan ${ }^{1}$, Naushad Ali ${ }^{1,5}$, Stan A. Lightfoot ${ }^{2,4}$, Panayotis Pantazis ${ }^{6}$, Chinthalapally V. Rao ${ }^{1,5}$, Russell \\ G. Postier ${ }^{3}$, Courtney W. Houchen ${ }^{1,4,5^{*}}$
}

1 Department of Medicine, the University of Oklahoma Health Sciences Center, Oklahoma City, Oklahoma, United States of America, 2 Department of Pathology, the University of Oklahoma Health Sciences Center, Oklahoma City, Oklahoma, United States of America, 3 Department of Surgery, the University of Oklahoma Health Sciences Center, Oklahoma City, Oklahoma, United States of America, 4 Department of Veterans Affairs Medical Center, Oklahoma City, Oklahoma, United States of America, 5 The Peggy and Charles Stephenson Cancer Center, Oklahoma City, Oklahoma, United States of America, 6 COARE Biotechnology Inc., Oklahoma City, Oklahoma, United States of America

\begin{abstract}
Stem cell pluripotency, angiogenesis and epithelial-mesenchymal transition (EMT) have been shown to be significantly upregulated in pancreatic ductal adenocarcinoma (PDAC) and many other aggressive cancers. The dysregulation of these processes is believed to play key roles in tumor initiation, progression, and metastasis, and is contributory to PDAC being the fourth leading cause of cancer-related deaths in the US. The tumor suppressor miRNA miR-145 downregulates critical pluripotency factors and oncogenes and results in repressed metastatic potential in PDAC. Additionally, the miR-200 family regulates several angiogenic factors which have been linked to metastasis in many solid tumors. We have previously demonstrated that downregulation of DCLK1 can upregulate critical miRNAs in both in vitro and in vivo cancer models and results in downregulation of c-MYC, KRAS, NOTCH1 and EMT-related transcription factors. A recent report has also shown that Dclk1 can distinguish between normal and tumor stem cells in $\mathrm{Apc}^{\mathrm{min} /+}$ mice and that ablation of Dclk1+ cells resulted in regression of intestinal polyps without affecting homeostasis. Here we demonstrate that the knockdown of DCLK1 using poly(lactide-co-glycolide)encapsulated-DCLK1-siRNA results in AsPC1 tumor growth arrest. Examination of xenograft tumors revealed, (a) increased miR-145 which results in decreased pluripotency maintenance factors OCT4, SOX2, NANOG, KLF4 as well as KRAS and RREB1; (b) increased let-7a which results in decreased pluripotency factor LIN28B; and (c) increased miR-200 which results in decreased VEGFR1, VEGFR2 and EMT-related transcription factors ZEB1, ZEB2, SNAIL and SLUG. Specificity of DCLK1 post-transcriptional regulation of the downstream targets of miR-145, miR-200 and let-7a was accomplished utilizing a luciferase-based reporter assay. We conclude that DCLK1 plays a significant master regulatory role in pancreatic tumorigenesis through the regulation of multiple tumor suppressor miRNAs and their downstream pro-tumorigenic pathways. This novel concept of targeting DCLK1 alone has several advantages over targeting single pathway or miRNA-based therapies for PDAC.
\end{abstract}

Citation: Sureban SM, May R, Qu D, Weygant N, Chandrakesan P, et al. (2013) DCLK1 Regulates Pluripotency and Angiogenic Factors via microRNADependent Mechanisms in Pancreatic Cancer. PLoS ONE 8(9): e73940. doi:10.1371/journal.pone.0073940

Editor: Chunming Liu, University of Kentucky, United States of America

Received April 22, 2013; Accepted July 23, 2013; Published September 9, 2013

Copyright: $\odot 2013$ Sureban et al. This is an open-access article distributed under the terms of the Creative Commons Attribution License, which permits unrestricted use, distribution, and reproduction in any medium, provided the original author and source are credited.

Funding: This work was supported by National Institutes of Health and National Cancer Institute grants: CA-137482 (CWH); Oklahoma Center for the Advancement of Science and Technology, and Oklahoma Center for Adult Stem Cell Research (CWH). The funders had no role in study design, data collection and analysis, decision to publish, or preparation of the manuscript.

Competing interests: $\mathrm{CWH}$ is a cofounder of COARE Biotechnology Inc., and this does not alter the authors' adherence to all the PLOS ONE policies and sharing data and materials.

*E-mail: courtney-houchen@ouhsc.edu

\section{Introduction}

Pancreatic ductal adenocarcinoma (PDAC) is the fourth leading cause of cancer-related deaths in the U.S. annually with a 5\% 5-year survival rate. Despite more than 10 years of FDA-approved therapeutic regimens and marked improvements in medical and surgical care, no significant impact on PDAC patient survival has been observed [1]. Recently, a subset of cells with cancer stem-cell (CSC) properties has been identified in PDAC [2] that are capable of unlimited self-renewal and give rise to more-differentiated and more-aggressive progeny, which are often resistant to 
conventional chemotherapy and radiotherapy [2,3]. The inability to eradicate these CSCs is postulated to be a reason for tumor relapse, metastasis and death following initial responses to treatment [4]. Another critical obstacle in combating solid tumor cancers in general is the heterogeneity of cell types within the tumor microenvironment [5] and the highly desmoplastic tumor niche [6]. This heterogeneity is further complicated by epithelial to mesenchymal transition (EMT), a process that plays a key role in cancer invasion and metastasis [7,8]. Many of the EMT-inducing transcription factors such as SNAI1 (SNAIL), SNAI2 (SLUG), ZEB1, ZEB2, TWIST1, FOXC2 and Goosecoid have been associated with tumor invasion and metastasis [9]. Recently, a number of reports have identified the microRNA (miRNA) miR-200 family as fundamental markers and regulators of EMT [10-12]. miRNAs are small, 19-22 nucleotide long, non-coding RNAs that inhibit gene expression at the posttranscriptional level [13]. A strong link between miRNA dysregulation and human cancer has been established [14]. Consequently miRNAs have been demonstrated to act either as oncogenes (e.g., miR-155, miR-17-5p and miR-21) [15,16] or tumor suppressors (e.g., miR-34, miR-15a, miR-16-1 and let-7) [17-20]. However, the precise regulatory features that tip the balance towards a cancer phenotype with respect to tumor suppressor versus oncogenic miRNA expression are poorly understood.

Pluripotency is the ability of a cell to differentiate into any cell type and is a unique characteristic of embryonic stem cells (ESCs). Pluripotency transcription factors such as OCT4, SOX2, NANOG and KLF4 form regulatory networks and influence a wide spectrum of downstream genes. Overexpression of these factors can dedifferentiate human and mouse somatic cells into induced pluripotent stem cells (iPSCs) [21]. The reprogramming factors OCT4, SOX2, NANOG, KLF4, C-MYC, and LIN28 have also been suggested to be oncogenes and may be implicated in the development of several cancers [21-26]. Multiple reports have shown that these transcription factors are regulated, at least in part, by miRNAs $[21,27,28]$. miR-145 specifically inhibits the aforementioned pluripotency factors by binding the 3' untranslated region (UTR) of mRNA, which leads to inhibition of ESCs, self-renewal, and induction of differentiation. Furthermore, loss of miR-145 impairs differentiation and elevates OCT4, SOX2, and KLF4 [21]. Additionally, it has been demonstrated that the miR-145 promoter is bound and repressed by OCT4 in ESCs. This indicates (a) the existence of a direct link between the core reprogramming factors and $m i R-145$, and (b) the presence of a double-negative feedback loop involving OCT4, SOX2, KLF4, and $m i R-145$ [21]. In cancer, miR-145 is downregulated and has been demonstrated to possess tumor suppressor properties. The loss of miR-145 (miR-143/145 cluster) is observed in KRAS mutated pancreatic cancers, and therapeutic restoration of these miRNAs abrogates tumorigenesis [29,30]. Furthermore, Ras-responsive element binding protein 1 (RREB1) represses miR-143/miR-145 promoter activity, which indicates that repression is an early event in pancreatic cancer initiation and progression [29]. Additionally, KRAS and RREB1 are targets of miR-143/145, demonstrating a feed-forward mechanism that potentiates RAS signaling-mediated PDAC tumor progression [29]. It has been recently demonstrated that ectopic expression of $m i R-143 / 145$ results in repressed metastasis and increased adhesion of pancreatic cancer cells [30]. These data taken together suggest that miR-145 is a master regulator of iPSCs factors in ESCs and CSCs and may play an important role in inhibition of pancreatic cancer initiation, progression and EMT.

Vascular endothelial growth factor (VEGF) signaling plays an important role in tumor angiogenesis. VEGF family members mediate the critical signaling by binding to two tyrosine kinase receptors, VEGFR1 and VEGFR2. VEGFR1 is required for endothelial cell survival; whereas, VEGFR2 is required for receptor-mediated angiogenic and vascular permeability activity [31-33]. Recently it has been demonstrated that these angiogenic factors (VEGFR1 and 2) are involved in tumor metastasis of renal and colon carcinoma cells [34]. VEGF signaling is known to promote tumor vasculature and endothelial cell proliferation in PDAC. Studies using soluble VEGFR1 and VEGFR2 (inhibition of VEGFR-mediated signaling in a dominant-negative manner) or the VEGFR tyrosine kinase inhibitors resulted in inhibition of tumor angiogenesis, growth and metastasis in PDAC mouse models [35-38].

DCLK1 (formerly known as DCAMKL-1) is a putative intestinal and pancreatic stem cell marker and is upregulated in the stroma and epithelium of PDAC. It has also recently been described as a marker of the relatively undefined tuft/brush cell in the pancreas and intestine $[39,40]$. It negatively regulates several tumor suppressor miRNAs and likely plays a key role in initiation and progression of solid tumor cancers [11,41]. In addition, it regulates several key oncogenes (c-MYC, KRAS and NOTCH1) and EMT. Moreover, overexpression of pluripotency factors in liver cancer cells resulted in increased expression of DCLK1 [42]. Recently Nakanishi et al. [43], have used an elegant Dclk1-Cre mouse model to demonstrate, that Dclk1 marks intestinal tumor stem cells. Ablation of Dclk1 expressing cells in $\mathrm{ApC}^{\mathrm{min} / \mathrm{+}}$ mice resulted in regression of polyps without any damage to the normal intestinal epithelium. In this report, following the knockdown of DCLK1 using nanoparticle-encapsulated siRNA (NPsiDCLK1) in tumor xenografted mice, we observed a significant increase in: (A) miR-143/145 cluster, which resulted in downregulation of key pluripotency markers (OCT4, SOX2, KLF4 and NANOG); (B) let-7a, which resulted in decreased pluripotency factor LIN28B; and (C) miR-200a, $b$ and $c$, which resulted in downregulation of EMT and angiogenic factors. Administration of NPsiDCLK1 did not result in overt toxicity in mice. These data taken together suggest a central regulatory role of DCLK1 in pancreatic tumorigenesis.

\section{Materials and Methods}

\section{Small interfering RNAs}

DCLK1 siRNA (siDCLK1) sequence targeting the coding region of DCLK1 (accession No. NM_004734) (GGGAGUGAGAACAAUCUACtt) and scrambled siRNAs (siSCR) not matching any of the human genes were obtained (Ambion Inc, Austin, TX). 


\section{Synthesis and characterization of DCLK1 siRNA NPs}

Poly(lactide-co-glycolide) acid nanoparticles (PLGA NPs) were synthesized using a double emulsion solvent evaporation technique as described earlier [11,44]. Briefly, siRNA (DCLK1 or scrambled) was condensed on the cationic polymer poly(ethyleneimine) (PEI) to form an siRNA-PEI complex. This complex was added to PLGA in chloroform and vortexed and transferred to $2 \%$ polyvinyl alcohol. This emulsion was sonicated and allowed to evaporate overnight. The size, polydispersity index, and zeta-potential measurements of synthesized siRNA NPs were determined using diffraction light scattering (DLS) utilizing Zeta PALS (Brookhaven Instruments, Holtsville, NY).

\section{Xenograft tumor model}

NOD/SCID mice were purchased from the Jackson Laboratory (Bar Harbor, Maine) and housed in pathogen-free conditions. They were cared for in accordance with guidelines set forth by the American Association for Accreditation of Laboratory Animal Care and the U.S. Public Health Service Commissioned Corps' "Policy on Human Care and Use of Laboratory Animals." All studies were approved and supervised by the University of Oklahoma Health Sciences Center's Institutional Animal Care and Use Committee (IACUC). AsPC-1 cells $\left(1 \times 10^{7}\right)$ were injected subcutaneously into the flanks of 4- to 6-wk-old mice $(n=3)$. Tumors were measured using a caliper and the volume was calculated as (length $\times$ width $^{2}$ ) $\times$ 0.5 . The tumors were palpable 30 days after injection of cells. NPs were reconstituted in sterile normal saline and injected directly into the tumors. Each animal bearing the tumor was injected on days $30,33,36,39$, and 42 with one of the following preparations $-50 \mu \mathrm{l}(5 \mu \mathrm{M})$ of siRNA-NP preparation [NP alone (Control), NP-siScrambled (NPsiSCR), or NPsiDCLK1]. All mice were killed on day 45.

Immunohistochemistry, Western blot, real-time reverse Transcription-PCR, miRNA analysis, Invasion assay and luciferase reporter gene assay

These analyses were carried out as previously described $[11,41]$. Detailed descriptions are provided in the Supplementary section of Materials and Methods (Text S1).

\section{Results}

\section{RNA silencing of DCLK1 inhibits pancreatic cancer xenograft growth}

AsPC-1 human pancreatic cancer cells were injected subcutaneously into the flanks of NOD/SCID mice and tumors were allowed to develop for 30 days. NP encapsulated siRNAs (NPsiDCLK1 and NPsiSCR) were injected intratumorally. Nanoparticle encapsulation was performed to overcome the theoretical limitations of siRNA-based delivery [45]. The efficacy of this approach has been previously tested in colorectal cancer xenografts [11]. On day 30, when the tumors were palpable, the treatments were initiated and continued every third day for a total of five injections. Tumors were excised at day 45, and the tumor volumes are represented in
Figure 1. Administration of NPsiDCLK1 resulted in a significant $(\sim 85 \%)$ reduction $(p<0.01)$ in tumor volume compared with either the Control (NPs-alone) or NPsiSCR-treated tumors (Figure 1A and 1B). mRNA analysis demonstrated a significant downregulation $(p<0.01)$ of DCLK1 mRNA compared to Control or NPsiSCR treated tumors (Figure 1C). These data taken together demonstrate that inhibition of DCLK1 results in AsPC-1 tumor xenograft growth arrest.

\section{Knockdown of DCLK1 results in downregulation of pluripotency factors in pancreatic tumor xenografts via miR-145}

It has been demonstrated that OCT4, SOX2, C-MYC, LIN28, NANOG and KLF4 are required for ESC self-renewal and pluripotency and are upregulated in some aggressive cancers and in CSCs [23-26,28]. Overexpression of these factors can reprogram or dedifferentiate human and mouse somatic cells into iPSCs. Here we observed a significant $(p<0.01)$ downregulation $(>40 \%)$ in the mRNA expression of pluripotency markers NANOG and KLF4 (Figure 2A and 2B) by real-time RT-PCR following the knockdown of DCLK1. Similarly NANOG and KLF4 proteins were also downregulated as analyzed by immunohistochemical analysis (Figure $2 \mathrm{C}$ and 2D). Additionally, we also observed a significant $(>40 \%)$ downregulation of OCT4 (Figure 2E) and SOX2 (Figure 2F) at the mRNA level following the knockdown of DCLK1 in AsPC-1 tumor xenografts.

\section{DCLK1 post-transcriptionally regulates miR-145 in pancreatic cancer}

miR-145 has been shown to repress OCT4, SOX2, and KLF4, thereby repressing pluripotency and controlling differentiation [21]. miR-145 is downregulated in various cancers and has been demonstrated to possess tumor suppressor and metastasis inhibitory properties [29,30]. Earlier reports $[11,28,41]$ and the data presented above indicate that DCLK1 negatively regulates tumor suppressor miRNAs like let-7a, miR-144 and miR-200a. Similarly, here we observed a significant induction (1.5-fold) of pri-miR-143/145 cluster miRNA (Figure 3A) and pri-miR-145 miRNA (Figure 3B) following the knockdown of DCLK1 in AsPC-1 tumor xenografts. Furthermore, we performed a luciferase reporter gene assay to quantitatively measure the effect of DCLK1 knockdown on miR-145 miRNA. AsPC-1 cells were transfected with a plasmid containing the firefly luciferase gene with a complementary miR-145 binding site at the 3' UTR. Following transfection, cells were treated with NPs alone, NPsiSCR, or NPsiDCLK1 and were subjected to luciferase activity measurement. A reduction in luciferase activity was observed in cells treated with NPsiDCLK1 compared to the control or NPsiSCR (Figure 3C). These data suggest that knockdown of DCLK1 results in downregulation of miR-145 miRNA downstream targets in pancreatic cancer cells. It has been previously demonstrated that a feedback loop mechanism exists between miR-143/145 and KRAS and RREB1. RREB1 is known to repress the transcription of $m i R-143 / 145$ by binding to its promoter region. Additionally, KRAS and RREB1 are downstream targets of miR-143/145 [29]. Knockdown of 

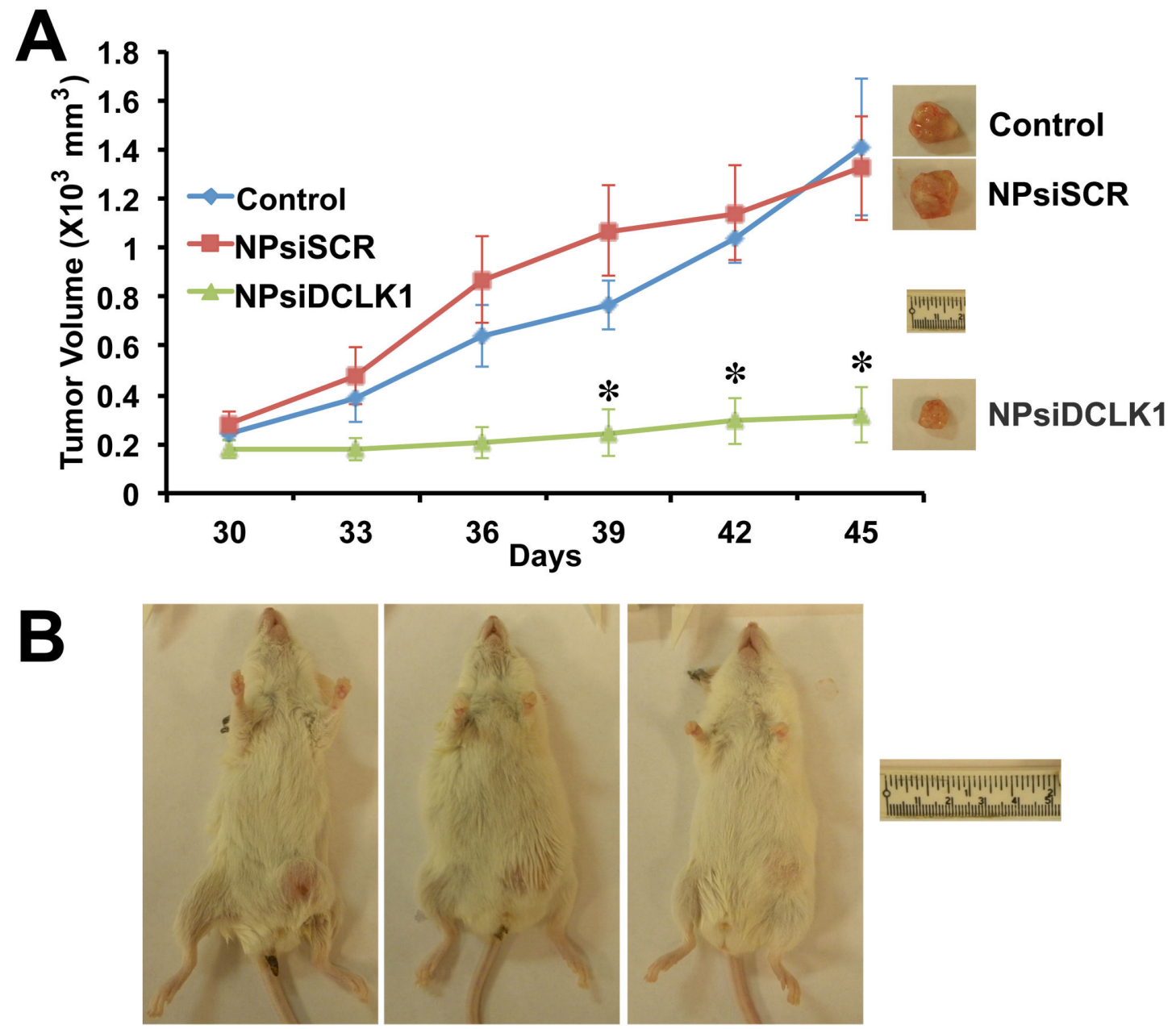

\section{Control NPsiSCR NPsiDCLK1}

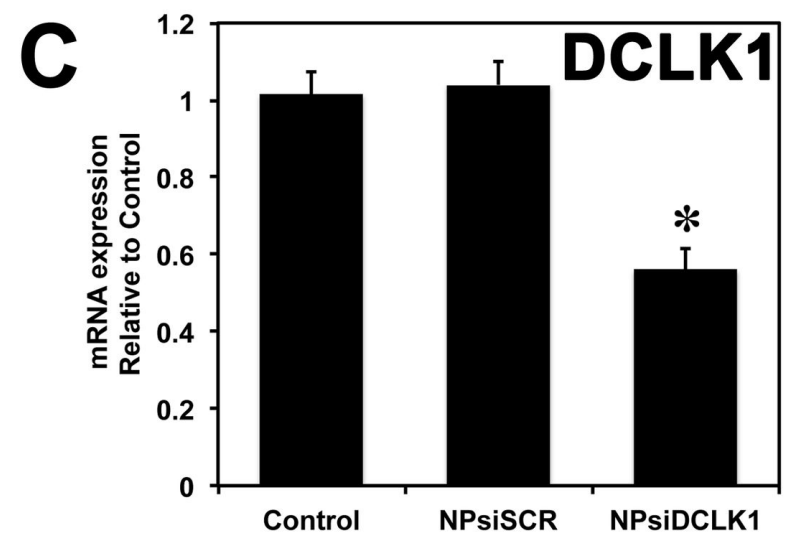

\section{Figure-1}

Figure 1. DCLK1 is essential for human pancreatic tumor xenograft growth. A, AsPC-1 human pancreatic cancer cells were subcutaneously injected into the flanks of NOD/SCID mice to generate tumors. At day 30, PLGA NP encapsulated siRNAs (siDCLK1 and siSCR) were injected directly into the tumors and followed by injections every third day. After 5 injections, tumors were excised at day 45 and are represented above. Tumor volume was measured every 3 days. B, Representative photograph of mice bearing the tumors from each group are shown. C, The expression of DCLK1 mRNA in the tumors quantitated by real-time RTPCR. Values are given as average \pm SEM, and asterisks denote statistically significant differences $\left({ }^{*} p<0.01\right)$ compared with Control (NP alone).

doi: 10.1371/journal.pone.0073940.g001 

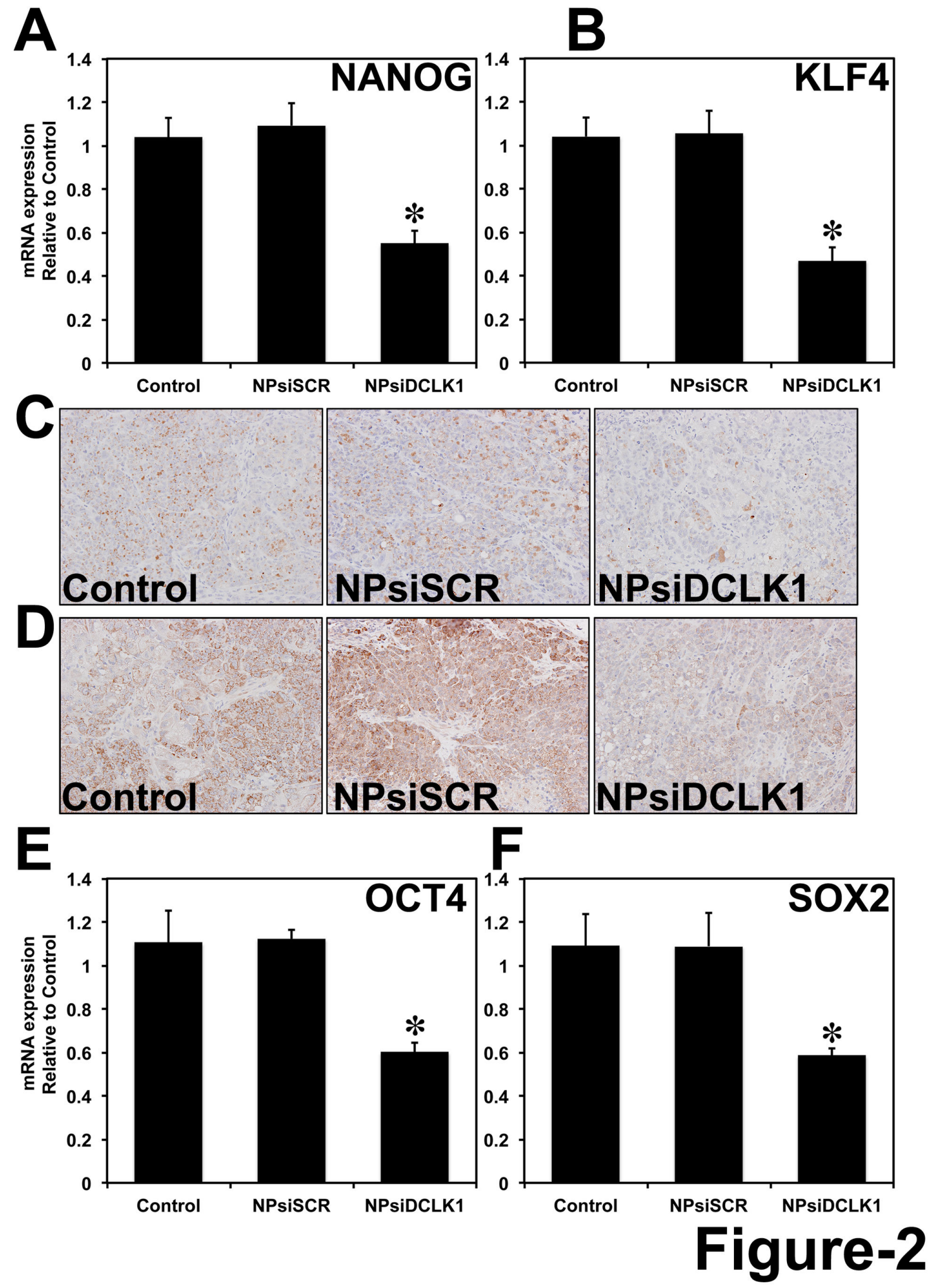

Figure 2. DCLK1 regulates pluripotency. siRNA-mediated knockdown of DCLK1 resulted in downregulation of pluripotency factors: NANOG mRNA (A); KLF4 mRNA (B); NANOG protein (C); KLF4 protein (D); OCT4 mRNA (E) and SOX-2 mRNA (F). mRNA was analyzed using real-time RT-PCR and protein by immunohistochemical analyses. For bar graph in $A, B, E$ and $F$, values are given as average \pm SEM, and asterisks denote statistically significant differences $\left({ }^{*} p<0.01\right)$ compared with Control (NP alone) . doi: 10.1371/journal.pone.0073940.g002 
DCLK1 resulted in increased expression of $m i R-143 / 145$ cluster and downregulation of its downstream target KRAS (Figure 3D). Furthermore, we found that the expression of RREB1 was significantly downregulated following the administration of siRNA against DCLK1 (Figure 3E). With these data, we speculate that DCLK1 plays a role in posttranscriptional regulation of $m i R-143 / 145$ cluster and thereby downregulates KRAS and RREB1 in pancreatic tumor xenografts.

Overall, these data taken together demonstrate a potential regulatory role for DCLK1 in the expression of iPSC factors in pancreatic cancers via miR-145 miRNA.

\section{DCLK1 regulates let-7a and its downstream target LIN28B in pancreatic cancer}

In pancreatic and colorectal cancer cells, we have previously demonstrated that DCLK1 negatively regulates miRNA let-7a. siRNA-mediated knockdown of DCLK1 resulted in downregulation of let-7a downstream targets c-MYC and KRAS. In pancreatic tumor xenografts treated with NPsiDCLK1, we observed a significant upregulation of let-7a (Figure 4A) and subsequent downregulation of its downstream target c-MYC (mRNA and protein) (Figure S1). Reports suggests that LIN28B, a pluripotency maintenance factor regulates miRNA let-7 and in turn let-7 regulates LIN28B (due to the presence of binding site in the 3' UTR of LIN28B), which suggests a double negative feedback loop between LIN28 and let-7 $[46,47]$. Here we wanted to see whether NPsiDCLK1 regulates downstream targets of let-7a miRNA. AsPC-1 cells were transfected with plasmid encoding firefly luciferase gene under the control of 3' UTR containing binding site for let-7. Following the transfection, the cells were treated with NPsiDCLK1 and subjected to luciferase measurement. Following the knockdown of DCLK1, we observed a significant downregulation of let-7-dependent luciferase activity (Figure 4B) indicating that NPsiDCLK1 regulates downstream targets of let-7 in pancreatic cancer cells. Based on real-time RT-PCR analysis of tumors treated with NPsiDCLK1, we observed a significant downregulation of LIN28B mRNA compared to NPsiSCR or control-treated tumors (Figure 4C). These data indicate that DCLK1 regulates LIN28B via let-7-dependent mechanism.

\section{DCLK1 regulates miR-200 family genes and EMT in pancreatic cancer}

EMT is a highly conserved process, characterized by the phenotypic conversion of epithelial cells to mesenchymal cells [7]. EMT is essential in various process including organ morphogenesis, wound healing, cancer metastasis and tissue remodeling in embryonic development. Recent studies have demonstrated that miR-200a, $b$ and $c(m i R-200)$ are known to regulate EMT and angiogenesis [48]. Previous studies have demonstrated that DCLK1 is overexpressed in human pancreatic cancer tissues and co-localizes with SNAIL and SLUG. Following the knockdown of DCLK1, a significant downregulation of ZEB1, ZEB2, SNAIL and SLUG was observed following increased expression of pri-miR-200a in AsPC-1 cancer cells [11]. Similarly in AsPC-1 tumor xenografts, a 2-fold induction of pri-miR-200a $(p<0.01)$ (Figure $5 A$ ) was observed. Furthermore, we wanted to investigate the effect of DCLK1 knockdown on expression of miR-200b and $c$ in AsPC-1 tumor xenografts. Similar to miR-200a, we observed a significant upregulation of $m i R-200 b$ (1.5-fold) (Figure $5 \mathrm{~A}$ ) and $m i R-200 c$ (2-fold) (Figure $5 \mathrm{~A}$ ) following the knockdown of DCLK1. Next, we wanted to investigate whether DCLK1 regulates the downstream targets of miR-200. We performed a luciferase reporter assay for miR-200. AsPC-1 cells were transfected separately with plasmids encoding the luciferase gene under the regulation of miRNA binding sites (three plasmids each containing binding sites for miR-200a, $b$ or $c$ ) at its 3' UTR. Following transfection, we treated the cells with NPsiDCLK1. The cell lysates were subjected to luciferase measurement. Following the knockdown of DCLK1, there was a significant downregulation of miR-200a, miR-200b and miR-200c (Figure 5B) mediated luciferase activity. These data indicate that DCLK1 regulates miR-200 and its downstream targets in PDAC. We also observed a subsequent reduction of miR-200 downstream targets ZEB1 and ZEB2 (Figure 5C), SNAIL and SLUG (Figure 5D) following the knockdown of DCLK1 in pancreatic tumor xenografts. Furthermore AsPC-1 cells were treated with NPsiSCR or NPsiDCLK1 and subjected to invasion assay using $\mathrm{BD}$ Biosciences Matrigel invasion chambers (BD BioCoat ${ }^{\mathrm{TM}}$ ). We observed significant inhibition of invasion following the knockdown of DCLK1 (Figure 5E and F). These data taken together indicate that knockdown of DCLK1 inhibits EMT and invasion by regulating miR-200 in human pancreatic tumor xenografts and cancer cells. Similarly to our previous studies, following the knockdown of DCLK1, we also observed inhibition of NOTCH1 via miR-144 (Figure S2) in AsPC-1 tumor xenografts.

\section{DCLK1 regulates angiogenic factors in PDAC}

It has been demonstrated that miR-200 inhibits lung adenocarcinoma invasion and metastasis by targeting VEGFR1. A putative binding site for miR-200 was observed in the 3' UTR of VEGFR1, and it was demonstrated that miR-200 negatively regulates VEGFR1 [48]. Additionally, a recent study demonstrated that VEGFR1 and VEGFR2 has a binding site for $m i R-200 b$ and based on luciferase-based reporter assay miR-200b regulates these angiogenic factors [49]. These data indicate that VEGFR1 and VEGFR2 are downstream targets of $m i R-200$. Here, we found DCLK1 regulating miR-200 and its downstream targets. We wanted to further investigate the effect of DCLK1 knockdown on angiogenic factors VEGFR1 and VEGFR2. We observed a significant downregulation of VEGFR1 mRNA (Figure 6A), protein (Figure 6B and C - left panel) in tumors treated with NPsiDCLK1 compared to control and NPsiSCR treated-tumors. We also observed downregulation of VEGFR2 mRNA (Figure 6E) and protein (Figure 6C - right panel) in pancreatic tumor xenografts treated with NPsiDCLK1. Additionally, we performed luciferase-based reporter assays for VEGFR1 and VEGFR2. AsPC-1 cells were transfected with luciferase gene with VEGFR1 or VEGFR2 3' UTR, treated with NPsiDCLK1 and subjected to luciferase activity measurement. Following the knockdown of DCLK1, we observed a significant downregulation of VEGFR1 (Figure 6D) 

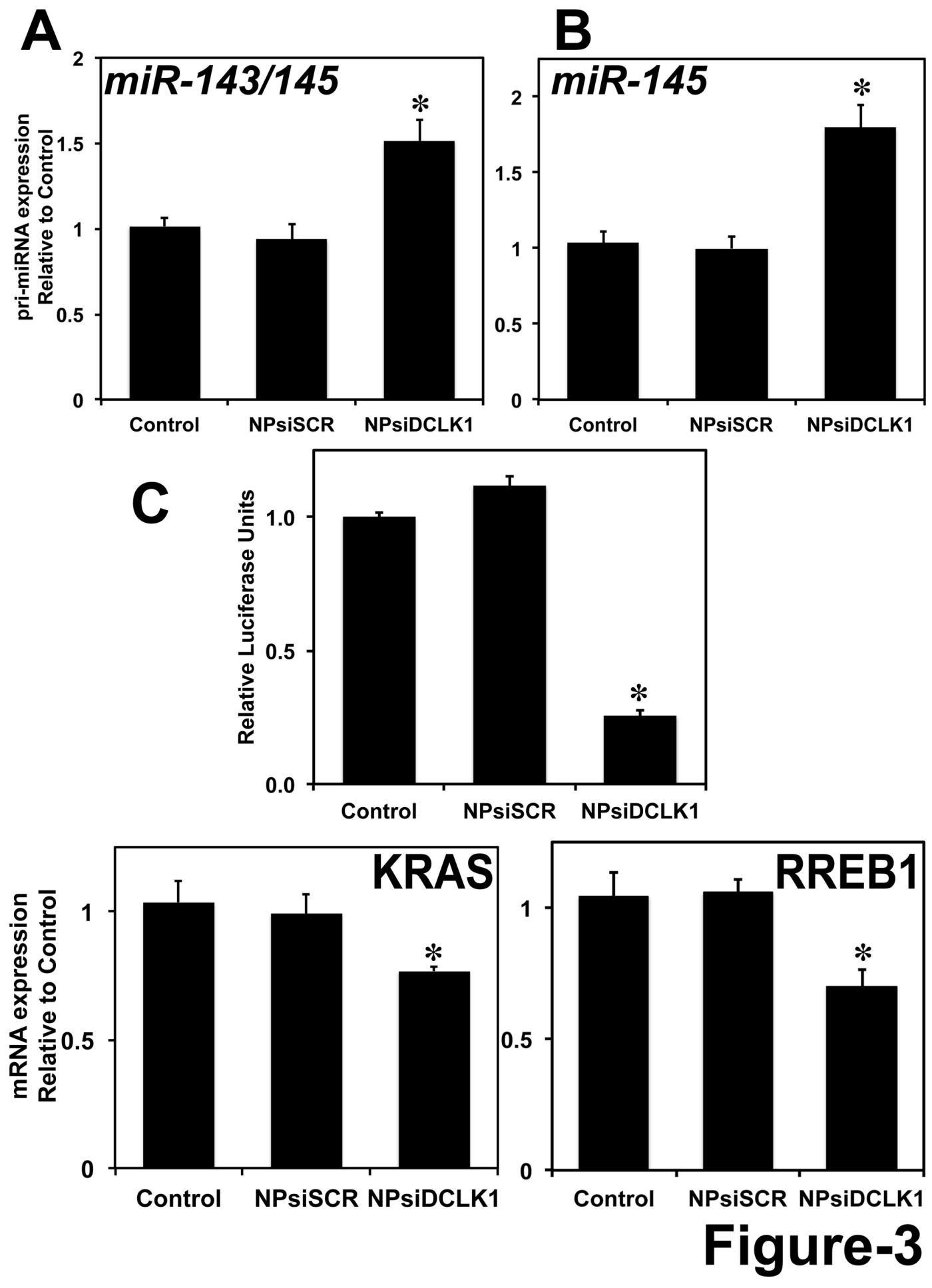

Figure 3. DCLK1 negatively regulates miR-143/145. Following the knockdown of DCLK1 in AsPC-1 tumor xenografts, a significant upregulation of miR-143/145 cluster (A) and miR-145 miRNA (B) by real-time RT-PCR. C, A decrease in luciferase activity (luciferase units) following transfection with plasmid-encoding luciferase containing the miR-145 binding site was observed following the knockdown of DCLK1 in AsPC-1 human pancreatic cancer cells. Knockdown of DCLK1 also resulted in the downregulation of KRAS (D) and RREB1 (E) mRNA, downstream targets of miR-143/145 miRNA cluster, analyzed using real-time RT-PCR. Values are given as average \pm SEM, and asterisks denote statistically significant differences $\left({ }^{*} p<0.01\right)$ compared with Control (NP alone).

doi: 10.1371/journal.pone.0073940.g003 

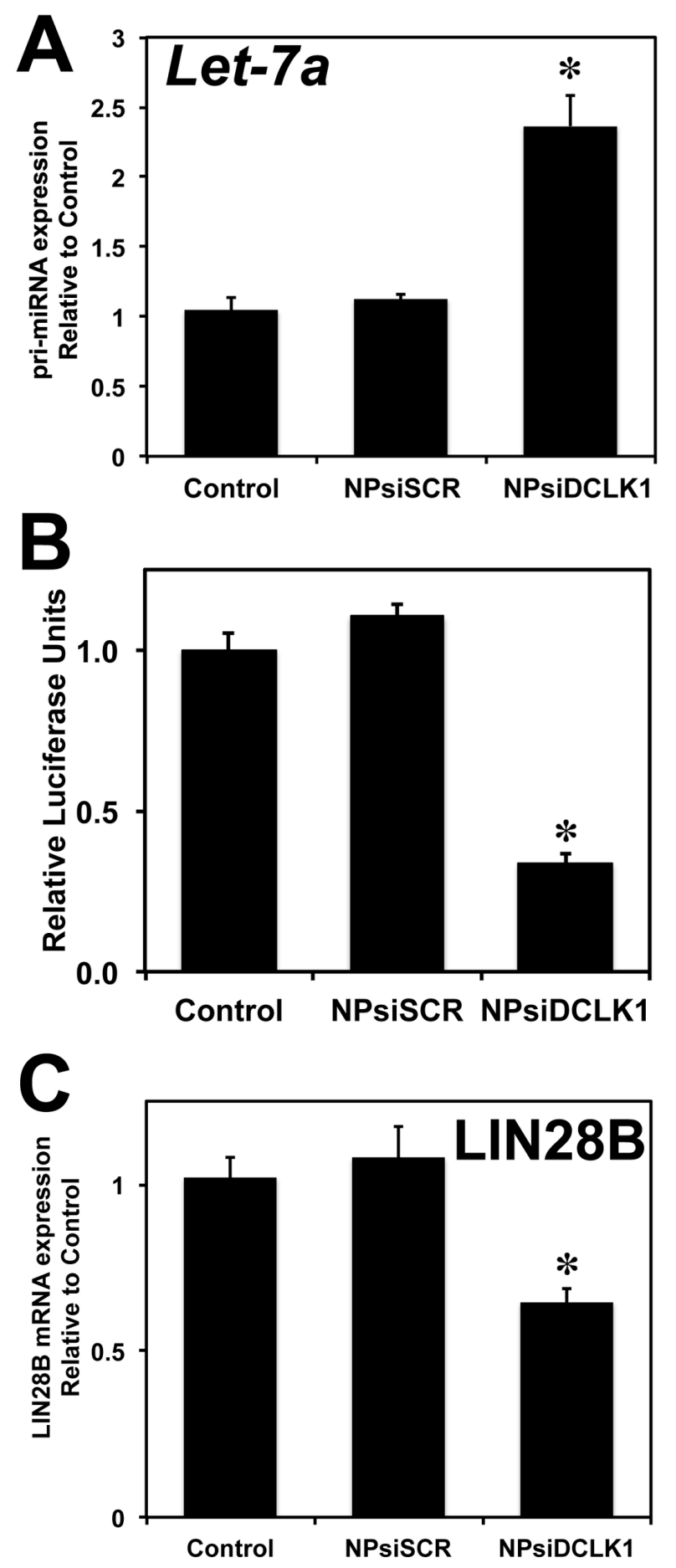

\section{Figure-4}

Figure 4. DCLK1 regulates LIN28B via let-7a. A, siRNA-mediated knockdown of DCLK1 in tumor xenografts results in increased expression of pri-let-7a miRNA. B, Following the knockdown of DCLK1, a decrease in miR-let7a dependent luciferase activity was observed in AsPC-1 cells. C, LIN28B mRNA downstream target of let-7a was downregulated in tumors treated with NPsiDCLK1. Values in the bar graphs are given as average \pm SEM, and asterisks denote statistically significant differences $\left({ }^{\star} P<0.01\right)$ compared with Control (NP alone).

doi: 10.1371/journal.pone.0073940.g004 

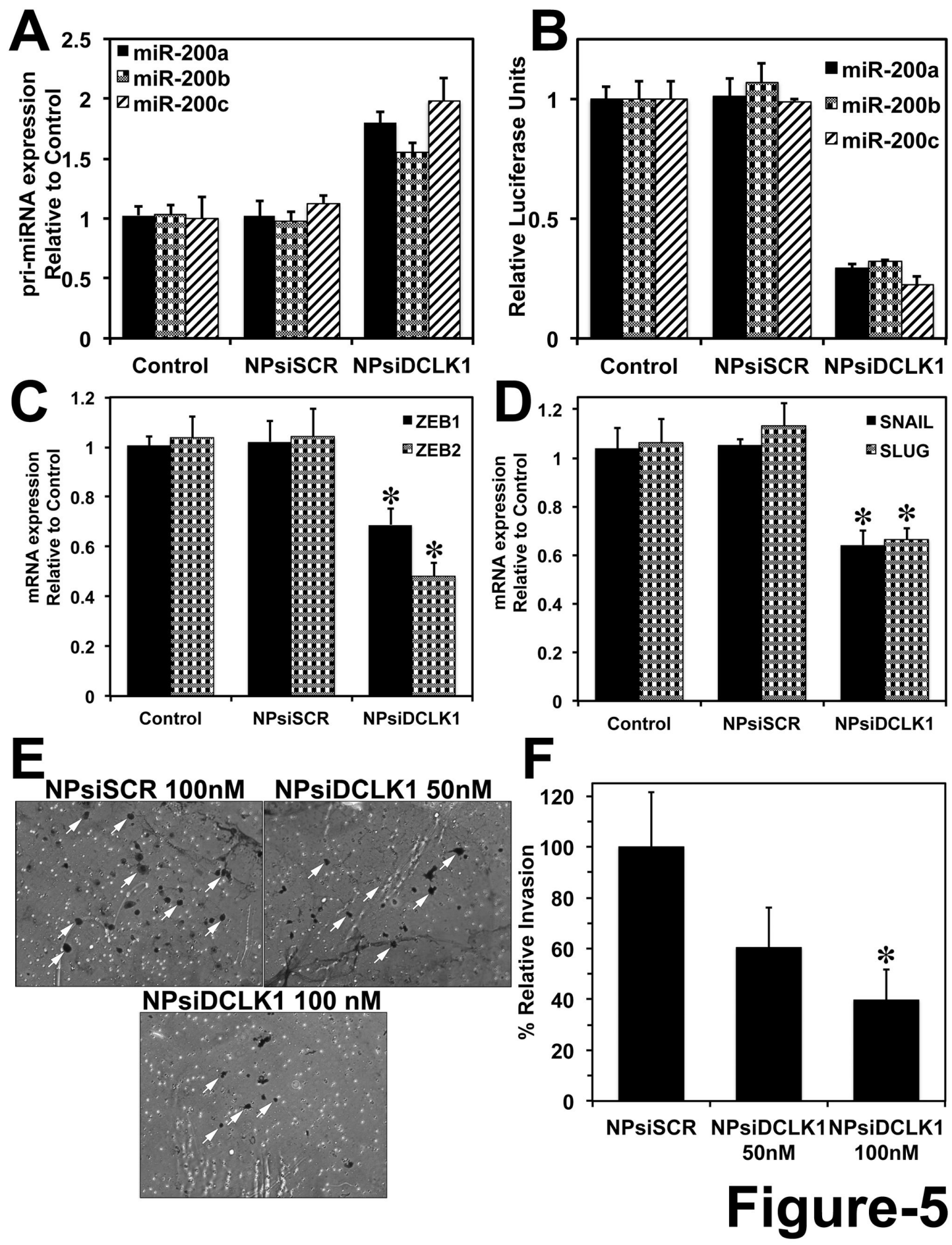

Figure 5. DCLK1 negatively regulates $m i R-200$ and inhibits EMT and invasion. A, siRNA-mediated knockdown of DCLK1 results in increased expression of pri-miR-200a, pri-miR-200b and pri-miR-200c by real-time RT-PCR. B, Following the knockdown of DCLK1, a decrease in miR-200a, miR-200b and miR-200c dependent luciferase activity was observed in AsPC-1 cells. Tumor xenografts treated with NPsiDCLK1 demonstrated a downregulation of EMT transcription factors ZEB1 and ZEB2 mRNA (C), decreased SNAIL and SLUG mRNA expression (D). E, siRNA-mediated inhibition of DCLK1 results in inhibition of invasion in AsPC-1 cells. White arrows indicate the cells invaded through the Matrigel matrix. F, Bar graph represents quantification of invaded cells following each treatment. Cells were counted in 5 different fields for each insert. Values in the bar graphs are given as average \pm SEM, and asterisks denote statistically significant differences $\left({ }^{*} P<0.01\right)$ compared with Control (NP alone).

doi: 10.1371/journal.pone.0073940.g005 
and VEGFR2 (Figure 6F) 3' UTR mediated luciferase activity. These data taken together indicate that DCLK1 regulates VEGFR1 and VEGFR2 via miR-200 in PDAC.

\section{Discussion}

miRNAs are rapidly emerging as critical regulators of virtually all key cellular processes. Dysregulation of miRNAs are quite common in many human cancers including PDAC. In many tumors, there is either overexpression of so-called oncogenic miRNAs (e.g., miR-155, miR-17-5p and miR-21) [15,16] or downregulation of tumor suppressor miRNAs (e.g., miR-34, miR-15a, miR-16-1 and let-7) [17-20]. The let-7 and miR-200 families are well-known regulators of key differentiation programs during development. Loss of let-7 in cancer results in progression and dedifferentiation, and the miR-200 family has been shown to be a key regulator of EMT. Furthermore, recent studies have linked let-7 with stem cell maintenance and EMT. Thus it is quite possible that tumor progression may represent a process that results in progressive dedifferentiation (EMT) towards a cell type that has a stem cell-like phenotype. Moreover, this process appears to be tightly regulated by miRNA-dependent mechanisms $[10,11,27,30]$. DCLK1 regulates EMT in human pancreatic cancer cells via a miR-200a dependent mechanism [11] and is also a regulator of let-7a in pancreatic and colorectal cancer cells, which supports the concept that these miRNAs are relevant and novel targets in several solid tumor cancers $[10,11,27,30,41]$. DCLK1 is a putative marker of intestinal and pancreatic stem cells and is upregulated in several solid tumors providing additional evidence of its potential key role in the tumorigenic process. In this report, we have demonstrated that in addition to regulating several tumor suppressor miRNAs and downstream oncogenic targets, DCLK1 inhibition results in upregulation of miRNAs that negatively regulate several key pluripotency and proangiogenic factors. These data support the notion that the DCLK1 is a central regulator of the tumor process.

Repression of miR-143 and miR-145, two co-transcribed miRNAs located on human chromosome $5 q$, has been reported in pancreatic cancer. Accumulating data suggest that they possess tumor suppressor activity [50,51]. Reduced miR-143/145 expression is a common feature of many tumor types including colorectal carcinoma and PDAC [29,50,51]. Moreover, expression of these miRNAs inhibits proliferation and activates apoptosis of cancer cells in vitro and in vivo [29]. The miR-143/145 cluster, cooperate and inhibit the expression of KRAS2 and its downstream effector, RREB1 [29]. It has been recently demonstrated that systemic delivery of miR-143/145 via nanovectors blocked the growth of MiaPaCa-2 derived subcutaneous xenografts compared with vehicle or mock nanovector empty plasmid controls [29,52]. The miRNA restitution was confirmed in treated xenografts by significant upregulation of the corresponding miRNA and significant decreases in specific miRNA targets (KRAS2 and RREB1 for $m i R-143 / 145)$. Here, we observed downregulation of c-MYC and KRAS via let-7a in AsPC1 tumor xenografts following the knockdown of DCLK1 (Figure S1 and Figure 3D) (a similar mechanism was previously demonstrated in pancreatic cancer cells).

Epidermal growth factor receptor (EGFR) is upregulated in various cancer including pancreatic [53]. Additionally, inhibition of EGF signaling leads to inhibition of cancer initiation and progression [54]. Recently, it was reported that miR-145 inhibits cell proliferation of human lung adenocarcinoma by targeting EGFR, indicating that miR-145 is a tumor suppressor miRNA [55]. Furthermore, in another published work, it was demonstrated that EGFR suppress miR-143 and miR-145 in a murine models of colon cancer [56]. These data taken together indicate that there is a negative feedback loop mechanism between EFGR and miR-143/145 similar to KRAS/RREB1 and miR-143/145. Following the knockdown of DCLK1, we observed increase expression miR-143/145 and significant downregulation of KRAS and RREB1. We expect EGF signaling will also be downregulated following the knockdown of DCLK1.

VEGF and its receptors VEGFR1 and VEGFR2 have been demonstrated to play an important role in tumor vascular growth, angiogenesis, and metastasis [34]. Angiogenic factors are upregulated in various cancers such as colorectal, breast, renal, liver, and ovarian and have been correlated with poor prognosis. Though PDAC is not a grossly vascular tumor, they exhibit endothelial cell proliferation, one of the mechanisms that increase angiogenesis [38]. Inhibition of VEGF-A, VEGFR1 and VEGFR2 resulted in inhibition of tumor growth and angiogenesis in mouse models of PDAC [35-38]. Based on previous studies and computational analysis of the 3' UTR of VEGFR1 and VEGFR2, we observed a putative binding site for miR-200 (miR-200a, $b$ and $c$ ) [48,49]. Following the knockdown of DCLK1, miR-200 miRNAs were upregulated and resulted in inhibition of its downstream targets (EMT-transcription and angiogenic factors). Taken together, knockdown of DCLK1 is beneficial in preventing EMT, metastasis and angiogenesis.

These exciting new data illustrate the enormous potential of miRNA-based therapy. In this report, we attempt to advance this concept a bit further. Our data suggest that by downregulating DCLK1 expression in pancreatic cancer, we are directly inducing or facilitating upregulation of several endogenous miRNAs within the tumors. The role of DCLK1 in the regulation of pluripotency in a cancer context is novel and may present an exciting new target for anti-cancer based therapy. Using this candidate approach, we contend that endogenous upregulation of tumor suppressors may have an advantage over small molecule inhibitor-based approaches or even protein or miRNA replacement therapies. For example, a single therapeutic miRNA may regulate hundreds of critical pathways that drive tumorigenesis; whereas, the introduction of a single DCLK1 targeted agent may induce multiple therapeutic miRNAs. This mechanism may be relatively safe if recently published reports suggesting that quiescent stem cell populations are dispensable for normal homeostatic processes but are likely activated during geno/cytotoxic injury and neoplasia are correct $[39,57]$. Additionally, a recent study has demonstrated that ablation of Dclk1+ cells in $\mathrm{Apc}^{\mathrm{min} /+}$ mice resulted in regression of intestinal polyps without affecting the normal intestinal homeostasis [43]. These data provide the 

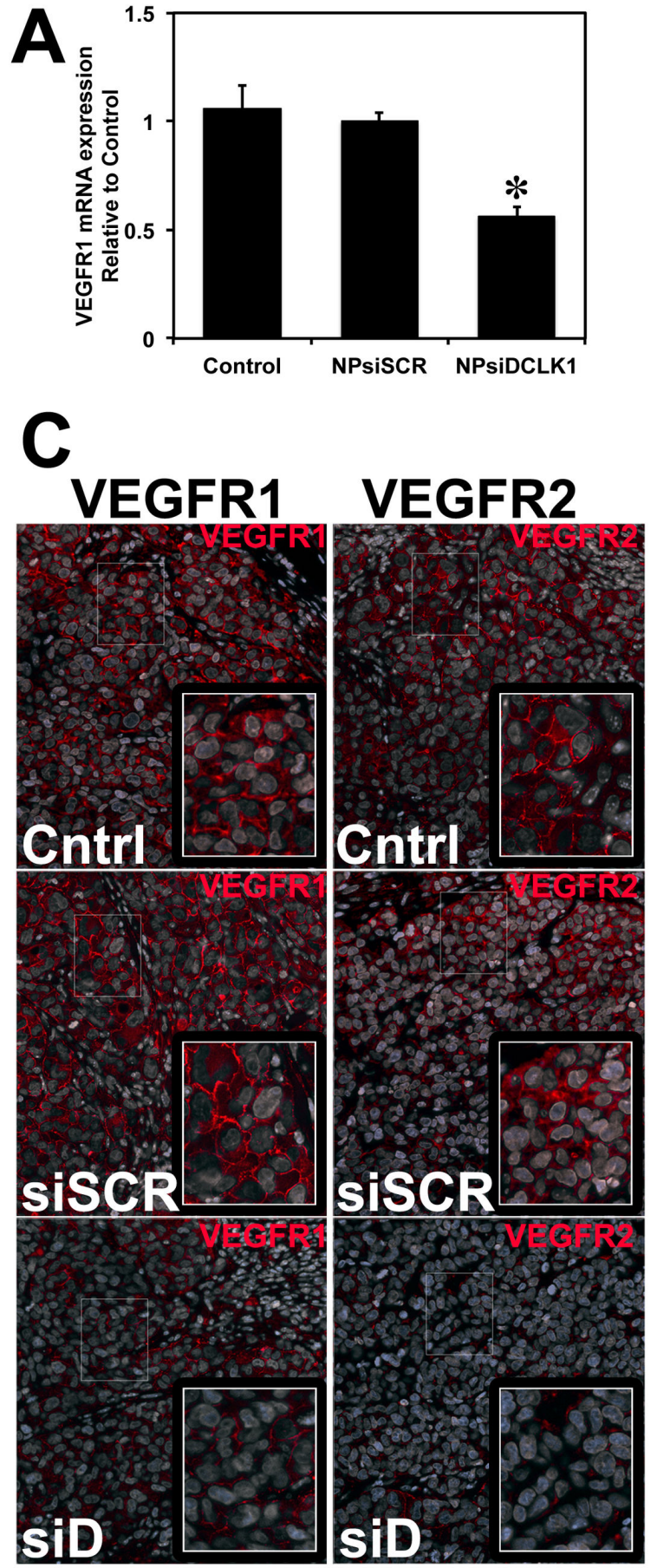

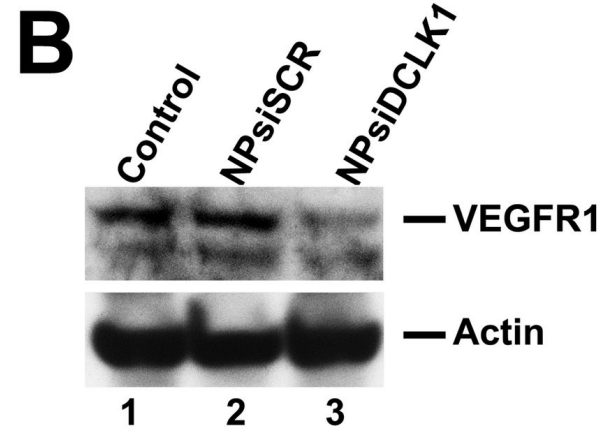

D
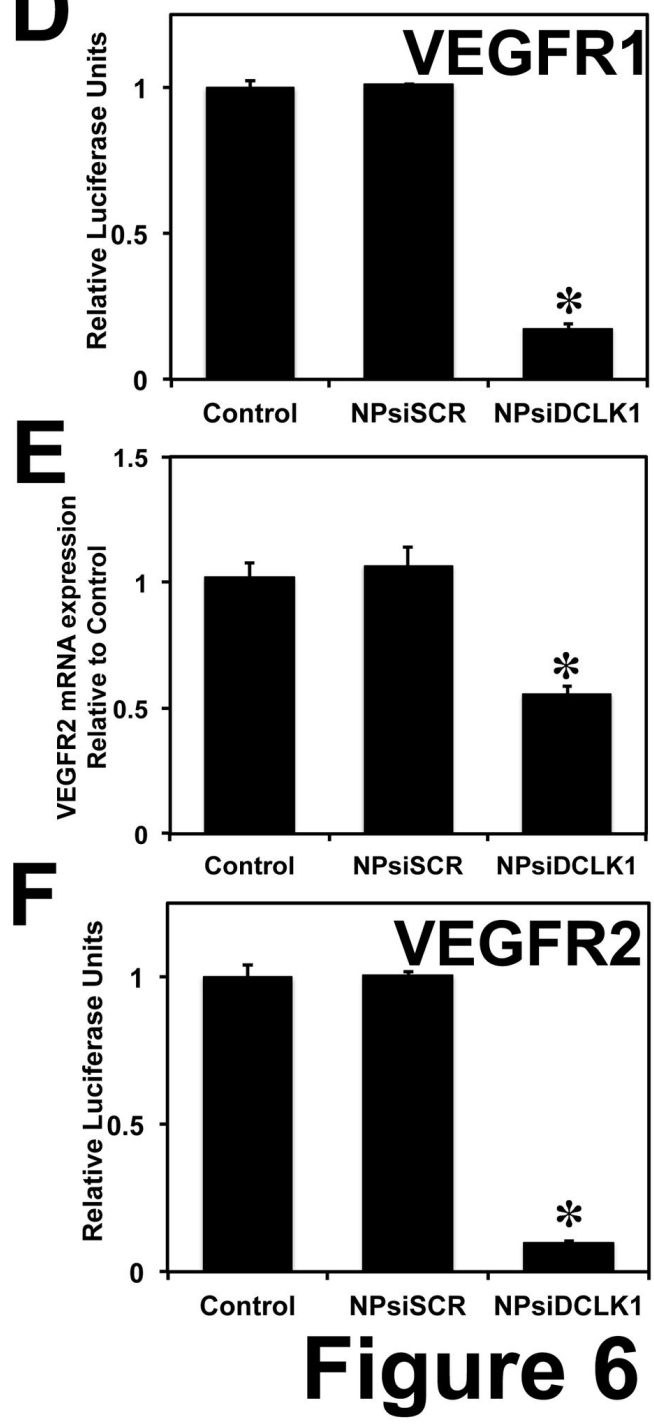

Figure 6. DCLK1 regulates angiogenic factors. A, siRNA-mediated knockdown of DCLK1 results in decreased expression of VEGFR1 mRNA in NPsiDCLK1 treated tumor xenografts. A decrease in VEGFR1 protein was observed in NPsiDCLK1 treated tumors (B - Western blot; C - Immunofluorescence - left panel, VEGFR1 red). D, Following the knockdown of DCLK1, a decrease in VEGFR1-3'UTR-dependent luciferase activity was observed in AsPC-1 cells. E, Knockdown of DCLK1 results in decreased expression of VEGFR2 mRNA in NPsiDCLK1 treated tumor xenografts. F, A decrease in VEGFR2-3'UTR-dependent luciferase activity was observed in AsPC-1 cells. NPsiDCLK1-mediated knockdown of DCLK1 resulted in decreased expression of VEGFR2 protein estimated using immunofluorescent analysis (C - right panel, VEGFR2 red). Values in the bar graphs in A, B, D and E, are given as average \pm SEM, and asterisks denote statistically significant differences $\left({ }^{*} P<0.01\right)$ compared with Control (NP alone).

doi: 10.1371/journal.pone.0073940.g006 
rationale for ongoing studies that investigate the role of DCLK1 in the regulation of miRNAs in cancer.

It has been demonstrated that process of dedifferentiation and EMT may result in generation of stem cell-like properties. Overexpression of pluripotency factors or stem cell maintaining factors results in dedifferentiation of fibroblasts to iPSCs. It has been recently reported that poorly differentiated breast, glioma and bladder cancer cells express a pluripotent stem cell-like signature [58]. Furthermore, induction of EMT in mammary epithelial cells generates or expresses stem cell-like phenotypes [9]. Cells isolated based on stem cell markers (CD44 ${ }^{\text {high }}$ and C24 ${ }^{\text {low }}$ ) had undergone EMT and overexpressed EMT markers like ZEB2, SNAIL and TWIST and also had the ability to form mammospheres $[9,27]$. These studies provide the link between the role of pluripotency factors and EMT in cancers that we explore in this report. Here we propose the hypothesis that DCLK1-mediated overexpression of these pluripotency factors in cancer cells may drive them towards a poorly differentiated phenotype, which may in turn facilitate the process of EMT [27]. Nevertheless, in this study, inhibition of DCLK1 results in inhibition of pluripotency markers and induction of miR-200 (EMT inhibitor) and thereby drives the cancer cells towards a differentiated state with reduced invasive properties. The inhibition of invasiveness of pancreatic cancer cells was observed in the invasion assay following the knockdown of DCLK1 (Figure 5E and F).

Overexpression of NOTCH1 led to the induction of EMT phenotype by activation of mesenchymal cell markers such as ZEB1, CD44, EpCAM, and Hes-1 [59]. Moreover, alteration of the miR-200 family has been found to be associated with the NOTCH signaling pathway in pancreatic CSCs. Furthermore, it was found that overexpression of miR-200 family significantly inhibits the NOTCH pathway in pancreatic cancer cells, which suggests the NOTCH pathway may be one of the miR-200 targets [60]. In this report, consistent with our previous studies, we observed downregulation of NOTCH1 in tumors treated with NPsiDCLK1 (Figure S2).

All of these studies indicate that pluripotency, angiogenesis, EMT, NOTCH1, and cancer stemness are all involved in the complex interplay of cellular signaling, which are critical features of the tumor microenvironment in pancreatic cancer that play an important role in cancer initiation, progression and metastasis. The studies presented clearly implicate DCLK1 in the regulation of $m i R-143 / 145, m i R-200$, EMT, pluripotency, angiogenesis, NOTCH1, and cancer stemness. Inhibition of multiple oncogenic pathways following the knockdown of DCLK1 was also observed in BxPC-3, human pancreatic cancer cell line (Figures S3 and S4). Finally, NPsiDCLK1 administered via intratumoral injection at a dose of $5 \mu \mathrm{M}$ for 15 days had no overt toxicity in the mice, which demonstrates its safety in vivo. These data taken together suggest that DCLK1 plays a central regulatory role in pancreatic tumorigenesis, and the delivery of targeted siRNA based therapeutics that inhibit multiple pro-tumorigenic pathways under the control of DCLK1 may represent a simple straightforward approach to combat pancreatic cancer and perhaps other human solid tumor cancers. Finally, we contend that beyond its status as a putative marker of quiescent stem cells and/or Tuft cells,
DCLK1 plays a central role in the initiation, progression and dissemination of cancer cells. This combined with its central regulatory role in stem cell-like processes, pluripotency factor regulation, EMT and angiogenesis makes it an ideal and novel target for therapy in pancreatic cancer and perhaps other solid tumors.

\section{Supporting Information}

Figure S1. siRNA-mediated knockdown of DCLK1 results in downregulation of C-MYC in pancreatic tumor xenografts. $A$ and $B, A$ decreased expression of $C-M Y C$ mRNA (using quantitative real-time RT-PCR) and protein (using Western blot) was observed in AsPC-1 tumor xenografts following knockdown of DCLK1. C, Decreased expression of cMYC protein (brown) was observed following the knockdown of DCLK1 by immunohistochemical analyses. For bar graph in A, values are given as average \pm SEM, and asterisks denote statistically significant differences $\left({ }^{*} p<0.01\right)$ compared with Control (NP alone).

(TIF)

Figure S2. NPsiRNA-mediated knockdown of DCLK1 downregulates NOTCH1 via miR-144. A, siRNA-mediated knockdown of DCLK1 decreases NOTCH1 mRNA in AsPC-1 tumor xenografts. B, A decrease in $\mathrm{NOTCH} 1$ protein by immunohistochemical analysis was also observed. $\mathrm{C}$, Knockdown of DCLK1 results in increased expression of primiR-144 miRNA in tumor xenografts. For bar graph in A and C, values are given as average \pm SEM, and asterisks denote statistically significant differences $\left({ }^{*} p<0.01\right)$ compared with Control (NP alone).

(TIF)

Figure S3. NPsiRNA-mediated knockdown of DCLK1 downregulates c-MYC via Let-7a, NOTCH1 via miR-144 and pluripotency factors via miR-143/145 in BxPC-3 cells. siRNA-mediated knockdown of DCLK1 in BxPC-3 results in decreased expression of DCLK1 mRNA (A), increased expression Let-7a, miR-144 and miR-143/145 (B), decreased expression of C-MYC and $\mathrm{NOTCH} 1$ (C), and decreased expression of NANOG, KLF4, OCT4 and SOX2 (D).

(TIF)

Figure S4. NPsiRNA-mediated knockdown of DCLK1 downregulates EMT transcription factors ZEB1, ZEB2 and angiogenic factors VEGFR1 and VEGFR2 via $m i R-200$ in BXPC-3 cells. siRNA-mediated knockdown of DCLK1 in BxPC-3 cells results in decreased expression of miR-200a, miR-200b and miR-200c (A), decreased expression of ZEB1 and ZEB2 (B), and decreased expression of VEGFR1 and VEGFR2 (C).

(TIF)

Text S1. Supporting methods. (DOCX) 


\section{Acknowledgements}

The authors thank Dr. llangovan Ramachandran for reviewing the manuscript, and Mrs. Sima Asfa for their technical assistance.

\section{References}

1. Jemal A, Siegel R, Ward E, Hao Y, Xu J et al. (2009) Cancer statistics, 2009. CA Cancer J Clin 59: 225-249. doi:10.3322/caac.20006. PubMed: 19474385.

2. Hermann PC, Huber SL, Herrler T, Aicher A, Ellwart JW et al. (2007) Distinct populations of cancer stem cells determine tumor growth and metastatic activity in human pancreatic cancer. Cell Stem Cell 1: 313-323. doi:10.1016/j.stem.2007.06.002. PubMed: 18371365.

3. Jimeno A, Feldmann G, Suárez-Gauthier A, Rasheed Z, Solomon A et al. (2009) A direct pancreatic cancer xenograft model as a platform for cancer stem cell therapeutic development. Mol Cancer Ther 8: 310-314. doi:10.1158/1535-7163.MCT-08-0924. PubMed: 19174553.

4. Li C, Heidt DG, Dalerba P, Burant CF, Zhang L et al. (2007) Identification of pancreatic cancer stem cells. Cancer Res 67: 1030-1037. doi:10.1158/0008-5472.CAN-06-2030. PubMed: 17283135.

5. Polyak K (2011) Heterogeneity in breast cancer. J Clin Invest 121: 3786-3788. doi:10.1172/JCl60534. PubMed: 21965334.

6. Hingorani SR, Wang L, Multani AS, Combs C, Deramaudt TB et al. (2005) Trp53R172H and KrasG12D cooperate to promote chromosomal instability and widely metastatic pancreatic ductal adenocarcinoma in mice. Cancer Cell 7: 469-483. doi:10.1016/j.ccr. 2005.04.023. PubMed: 15894267.

7. Turley EA, Veiseh M, Radisky DC, Bissell MJ (2008) Mechanisms of disease: epithelial-mesenchymal transition--does cellular plasticity fuel neoplastic progression? Nat Clin Pract Oncol 5: 280-290. doi:10.1038/ ncpcardio1163. PubMed: 18349857

8. Diehn M, Clarke MF (2006) Cancer stem cells and radiotherapy: new insights into tumor radioresistance. J Natl Cancer Inst 98: 1755-1757. doi:10.1093/jnci/djj505. PubMed: 17179471.

9. Mani SA, Guo W, Liao MJ, Eaton EN, Ayyanan A et al. (2008) The epithelial-mesenchymal transition generates cells with properties of stem cells. Cell 133: 704-715. doi:10.1016/j.cell.2008.03.027. PubMed: 18485877.

10. Park SM, Gaur AB, Lengyel E, Peter ME (2008) The miR-200 family determines the epithelial phenotype of cancer cells by targeting the Ecadherin repressors ZEB1 and ZEB2. Genes Dev 22: 894-907. doi: 10.1101/gad.1640608. PubMed: 18381893.

11. Sureban SM, May R, Lightfoot SA, Hoskins AB, Lerner M et al. (2011) DCAMKL-1 regulates epithelial-mesenchymal transition in human pancreatic cells through a miR-200a-dependent mechanism. Cancer Res 71: 2328-2338. doi:10.1158/0008-5472.CAN-10-2738. PubMed: 21285251

12. Sureban SM, May R, Mondalek FG, Qu D, Ponnurangam $S$ et al. (2011) Nanoparticle-based delivery of siDCAMKL-1 increases microRNA-144 and inhibits colorectal cancer tumor growth via a Notch-1 dependent mechanism. J Nanobiotechnol 9: 40. doi: 10.1186/1477-3155-9-40.

13. Cullen BR (2004) Transcription and processing of human microRNA precursors. Mol Cell 16: 861-865. doi:10.1016/j.molcel.2004.12.002. PubMed: 15610730.

14. Zhang L, Huang J, Yang N, Greshock J, Megraw MS et al. (2006) microRNAs exhibit high frequency genomic alterations in human cancer. Proc Natl Acad Sci U S A 103: 9136-9141. doi:10.1073/pnas. 0508889103. PubMed: 16754881.

15. He L, Thomson JM, Hemann MT, Hernando-Monge E, Mu D et al. (2005) A microRNA polycistron as a potential human oncogene. Nature 435: 828-833. doi:10.1038/nature03552. PubMed: 15944707.

16. Voorhoeve PM, le Sage C, Schrier M, Gillis AJ, Stoop H et al. (2006) A genetic screen implicates miRNA-372 and miRNA-373 as oncogenes in testicular germ cell tumors. Cell 124: 1169-1181. doi:10.1016/j.cell. 2006.02.037. PubMed: 16564011.

17. Calin GA, Dumitru CD, Shimizu M, Bichi R, Zupo S et al. (2002) Frequent deletions and down-regulation of micro- RNA genes miR15 and miR16 at $13 q 14$ in chronic lymphocytic leukemia. Proc Natl Acad Sci U S A 99: 15524-15529. doi:10.1073/pnas.242606799. PubMed: 12434020.

\section{Author Contributions}

Conceived and designed the experiments: SMS $\mathrm{CWH}$. Performed the experiments: SMS RM DQ NW PC. Analyzed the data: NA SAL PP CVR RGP. Contributed reagents/ materials/analysis tools: CWH. Wrote the manuscript: SMS.

18. Johnson SM, Grosshans H, Shingara J, Byrom M, Jarvis R et al. (2005) RAS is regulated by the let-7 microRNA family. Cell 120:635-647. doi: 10.1016/j.cell.2005.01.014. PubMed: 15766527.

19. Yanaihara N, Caplen N, Bowman E, Seike M, Kumamoto $K$ et al. (2006) Unique microRNA molecular profiles in lung cancer diagnosis and prognosis. Cancer Cell 9: 189-198. doi:10.1016/j.ccr.2006.01.025. PubMed: 16530703

20. Takamizawa J, Konishi $\mathrm{H}$, Yanagisawa $\mathrm{K}$, Tomida $\mathrm{S}$, Osada $\mathrm{H}$ et al. (2004) Reduced expression of the let-7 microRNAs in human lung cancers in association with shortened postoperative survival. Cancer Res 64: 3753-3756. doi:10.1158/0008-5472.CAN-04-0637. PubMed: 15172979.

21. Xu N, Papagiannakopoulos T, Pan G, Thomson JA, Kosik KS (2009) MicroRNA-145 regulates OCT4, SOX2, and KLF4 and represses pluripotency in human embryonic stem cells. Cell 137: 647-658. doi: 10.1016/j.cell.2009.02.038. PubMed: 19409607.

22. Shankar S, Nall D, Tang SN, Meeker D, Passarini J et al. (2011) Resveratrol inhibits pancreatic cancer stem cell characteristics in human and KrasG12D transgenic mice by inhibiting pluripotency maintaining factors and epithelial-mesenchymal transition. PLOS ONE 6: e16530. doi:10.1371/journal.pone.0016530. PubMed: 21304978.

23. Chen YC, Hsu HS, Chen YW, Tsai TH, How CK et al. (2008) Oct-4 expression maintained cancer stem-like properties in lung cancerderived CD133-positive cells. PLOS ONE 3: e2637. doi:10.1371/ journal.pone.0002637. PubMed: 18612434

24. Wen J, Park JY, Park KH, Chung HW, Bang S et al. (2010) Oct4 and Nanog expression is associated with early stages of pancreatic carcinogenesis. Pancreas 39: 622-626. doi:10.1097/MPA. Ob013e3181c75f5e. PubMed: 20173672.

25. Prasad NB, Biankin AV, Fukushima N, Maitra A, Dhara S et al. (2005) Gene expression profiles in pancreatic intraepithelial neoplasia reflect the effects of Hedgehog signaling on pancreatic ductal epithelial cells. Cancer Res 65: 1619-1626. doi:10.1158/0008-5472.CAN-04-1413. PubMed: 15753353

26. Wei D, Wang L, Kanai M, Jia Z, Le X et al. (2010) KLF4a up-regulation promotes cell cycle progression and reduces survival time of patients with pancreatic cancer. Gastroenterology 139: 2135-2145. doi:10.1053/ j.gastro.2010.08.022. PubMed: 20727893

27. Peter ME (2009) Let-7 and miR-200 microRNAs: guardians against pluripotency and cancer progression. Cell Cycle 8: 843-852. doi: 10.4161/cc.8.6.7907. PubMed: 19221491.

28. Sureban SM, Johnson JJ, Houchen CW (2012) Cancer Stem Cells and Pluripotency. Pancreatic Disorders and Therapy 02.

29. Kent OA, Chivukula RR, Mullendore M, Wentzel EA, Feldmann $G$ et al. (2010) Repression of the miR-143/145 cluster by oncogenic Ras initiates a tumor-promoting feed-forward pathway. Genes Dev 24: 2754-2759. doi:10.1101/gad.1950610. PubMed: 21159816.

30. Peng X, Guo W, Liu T, Wang X, Tu X et al. (2011) Identification of miRs-143 and -145 that is associated with bone metastasis of prostate cancer and involved in the regulation of EMT. PLOS ONE 6: e20341. doi:10.1371/journal.pone.0020341. PubMed: 21647377.

31. Yao J, Wu X, Zhuang G, Kasman IM, Vogt T et al. (2011) Expression of a functional VEGFR-1 in tumor cells is a major determinant of anti-PIGF antibodies efficacy. Proc Natl Acad Sci U S A 108: 11590-11595. doi: 10.1073/pnas.1109029108. PubMed: 21709213.

32. Bhattacharya R, Kang-Decker N, Hughes DA, Mukherjee $P$, Shah V et al. (2005) Regulatory role of dynamin-2 in VEGFR-2/KDR-mediated endothelial signaling. FASEB J 19: 1692-1694. PubMed: 16049137.

33. Cao $Y$ (2009) Positive and negative modulation of angiogenesis by VEGFR1 ligands. Sci Signal 2: re1 PubMed: 19244214

34. Lee YJ, Karl DL, Maduekwe UN, Rothrock C, Ryeom S et al. (2010) Differential effects of VEGFR-1 and VEGFR-2 inhibition on tumor metastases based on host organ environment. Cancer Res 70 : 8357-8367. doi:10.1158/0008-5472.CAN-10-1138. PubMed: 20978198.

35. Hoshida T, Sunamura M, Duda DG, Egawa S, Miyazaki S et al. (2002) Gene therapy for pancreatic cancer using an adenovirus vector encoding soluble flt-1 vascular endothelial growth factor receptor. 
Pancreas 25: 111-121. doi:10.1097/00006676-200208000-00001. PubMed: 12142732.

36. Ogawa T, Takayama K, Takakura N, Kitano S, Ueno H (2002) Antitumor angiogenesis therapy using soluble receptors: enhanced inhibition of tumor growth when soluble fibroblast growth factor receptor-1 is used with soluble vascular endothelial growth factor receptor. Cancer Gene Ther 9: 633-640. doi:10.1038/sj.cgt.7700478. PubMed: 12136423.

37. Solorzano CC, Baker CH, Bruns CJ, Killion JJ, Ellis LM et al. (2001) Inhibition of growth and metastasis of human pancreatic cancer growing in nude mice by PTK 787/ZK222584, an inhibitor of the vascular endothelial growth factor receptor tyrosine kinases. Cancer Biother Radiopharm 16: 359-370.

38. Korc M (2003) Pathways for aberrant angiogenesis in pancreatic cancer. Mol Cancer 2: 8. doi:10.1186/1476-4598-2-8. PubMed: 12556241.

39. Gerbe F, van Es JH, Makrini L, Brulin B, Mellitzer G et al. (2011) Distinct ATOH1 and Neurog3 requirements define tuft cells as a new secretory cell type in the intestinal epithelium. J Cell Biol 192: 767-780. doi:10.1083/jcb.201010127. PubMed: 21383077.

40. Bjerknes $M$, Khandanpour C, Möröy T, Fujiyama $T$, Hoshino $M$ et al. (2012) Origin of the brush cell lineage in the mouse intestinal epithelium. Dev Biol 362: 194-218. doi:10.1016/j.ydbio.2011.12.009. PubMed: 22185794.

41. Sureban SM, May R, Ramalingam S, Subramaniam D, Natarajan G et al. (2009) Selective blockade of DCAMKL-1 results in tumor growth arrest by a Let-7a MicroRNA-dependent mechanism. Gastroenterology 137: 649-659, e641-642 doi:10.1053/j.gastro.2009.05.004. PubMed: 19445940 .

42. Ali N, Allam H, May R, Sureban SM, Bronze MS et al. (2011) Hepatitis C virus-induced cancer stem cell-like signatures in cell culture and murine tumor xenografts. J Virol 85: 12292-12303. doi:10.1128/JVI. 05920-11. PubMed: 21937640

43. Nakanishi Y, Seno H, Fukuoka A, Ueo T, Yamaga Y et al. (2013) Dclk1 distinguishes between tumor and normal stem cells in the intestine. Nat Genet 45: 98-103. PubMed: 23202126.

44. Mondalek FG, Ponnurangam S, Govind J, Houchen C, Anant S et al. (2010) Inhibition of angiogenesis- and inflammation-inducing factors in human colon cancer cells in vitro and in ovo by free and nanoparticleencapsulated redox dye, DCPIP. J Nanobiotechnology 8: 17. doi: 10.1186/1477-3155-8-17. PubMed: 20633276.

45. de Fougerolles AR (2008) Delivery vehicles for small interfering RNA in vivo. Hum Gene Ther 19: 125-132. doi:10.1089/hum.2008.928. PubMed: 18257677.

46. Yang X, Lin X, Zhong X, Kaur S, Li N et al. (2010) Double-negative feedback loop between reprogramming factor LIN28 and microRNA let-7 regulates aldehyde dehydrogenase 1-positive cancer stem cells. Cancer Res 70: 9463-9472. doi:10.1158/0008-5472.CAN-10-2388. PubMed: 21045151.

47. Zhong X, Li N, Liang S, Huang Q, Coukos G et al. (2010) Identification of microRNAs regulating reprogramming factor LIN28 in embryonic stem cells and cancer cells. J Biol Chem 285: 41961-41971. doi: 10.1074/jbc.M110.169607. PubMed: 20947512.

48. Roybal JD, Zang Y, Ahn YH, Yang Y, Gibbons DL et al. (2011) miR-200 Inhibits lung adenocarcinoma cell invasion and metastasis by targeting Flt1/NEGFR1. Mol Cancer Res 9: 25-35. doi: 10.1158/1541-7786.MCR-10-0497. PubMed: 21115742.

49. Choi YC, Yoon S, Jeong Y, Yoon J, Baek K (2011) Regulation of vascular endothelial growth factor signaling by miR-200b. Mol Cells 32 : 77-82. doi:10.1007/s10059-011-1042-2. PubMed: 21544626.

50. Michael MZ, O' Connor SM, van Holst Pellekaan NG, Young GP, James RJ (2003) Reduced accumulation of specific microRNAs in colorectal neoplasia. Mol Cancer Res 1: 882-891. PubMed: 14573789.

51. Akao Y, Nakagawa Y, Kitade Y, Kinoshita T, Naoe T (2007) Downregulation of microRNAs-143 and -145 in B-cell malignancies. Cancer Sci 98: 1914-1920. doi:10.1111/j.1349-7006.2007.00618.x. PubMed: 17892514

52. Pramanik D, Campbell NR, Karikari C, Chivukula R, Kent OA et al. (2011) Restitution of tumor suppressor microRNAs using a systemic nanovector inhibits pancreatic cancer growth in mice. Mol Cancer Ther 10: 1470-1480. doi:10.1158/1535-7163.MCT-11-0152. PubMed: 21622730.

53. Perera RM, Bardeesy N (2012) Ready, Set, Go: The EGF Receptor at the Pancreatic Cancer Starting Line. Cancer Cell 22: 281-282. doi: 10.1016/j.ccr.2012.08.019. PubMed: 22975369.

54. Navas C, Hernández-Porras I, Schuhmacher AJ, Sibilia M, Guerra C et al. (2012) EGF Receptor Signaling Is Essential for K-Ras OncogeneDriven Pancreatic Ductal Adenocarcinoma. Cancer Cell 22: 318-330. doi:10.1016/j.ccr.2012.08.001. PubMed: 22975375.

55. Cho WC, Chow AS, Au JS (2011) MiR-145 inhibits cell proliferation of human lung adenocarcinoma by targeting EGFR and NUDT1. RNA Biol 8: 125-131. doi:10.4161/rna.8.1.14259. PubMed: 21289483.

56. Zhu H, Dougherty U, Robinson V, Mustafi R, Pekow J et al. (2011) EGFR signals downregulate tumor suppressors miR-143 and miR-145 in Western diet-promoted murine colon cancer: role of $\mathrm{G} 1$ regulators. Mol Cancer Res 9: 960-975. doi:10.1158/1541-7786.MCR-10-0531. PubMed: 21653642.

57. Tian H, Biehs B, Warming S, Leong KG, Rangell L et al. (2011) A reserve stem cell population in small intestine renders Lgr5-positive cells dispensable. Nature 478: 255-259. doi:10.1038/nature10408. PubMed: 21927002.

58. Ben-Porath I, Thomson MW, Carey VJ, Ge R, Bell GW et al. (2008) An embryonic stem cell-like gene expression signature in poorly differentiated aggressive human tumors. Nat Genet 40: 499-507. doi: 10.1038/ng.127. PubMed: 18443585.

59. Bao B, Wang Z, Ali S, Kong D, Li Y et al. (2011) Notch-1 induces epithelial-mesenchymal transition consistent with cancer stem cell phenotype in pancreatic cancer cells. Cancer Lett 307: 26-36. doi: 10.1016/j.canlet.2011.03.012. PubMed: 21463919.

60. Brabletz S, Bajdak K, Meidhof S, Burk U, Niedermann G et al. (2011) The ZEB1/miR-200 feedback loop controls Notch signalling in cancer cells. EMBO J 30: 770-782. doi:10.1038/emboj.2010.349. PubMed: 21224848. 